Z. klin. Chem. u. klin. Biochem.

8. Jg., S. $458-464$, September 1970

\title{
Über alkalische Phosphatasen in menschlichen Faeces
}

\author{
Von Vera Benić und Marijana Fišer-Herman \\ Klinik fïr Infektionskrankbeiten der Medizinischen Fakultät der Universität Ziagreb \\ (Vorstand: Prof. Dr. F. Mibaljević) \\ (Eingegangen am 16. März 1970)
}

Alkalische Phosphatasen (EC 3.1.3.1) in Faeces von Gesunden und Darmkranken wurden untersucht. Stärkegelelektrophorese, Messungen der Gesamtaktivität, der Hemmung durch L-Phenylalanin nach Frshman und Gose (7) und der Wärmeinaktivierung nach PosEN (6) zeigten, daß dic in den Exktementen vorhandenen alkalischen Phosphatasen z. T. aus der Dünndarmschleimhaut und z. T. aus der Galle stammen. Es könnte sein, daß auch noch Phosphatasen anderer Herkunft ausgeschieden werden.

Bei manchen Darmkrankheiten kommt es zu charakteristischen Änderungen in der Gesamtausscheidung und im Verhältnis zwischen den Enzymfraktionen. Kranke, welche an Enterocolitis litten, schieden im Stuhl viel mehr alkalische Phosphatase aus als Gesunde. Bei Protozoeninfektionen war die Ausscheidung herabgesetzt und die Eigenschaften der aus den Faeces extrahierten Phosphatasen glichen denen von Gallenphosphatasen.

Vergleichende Analysen weisen darauf hin, daß das in den Verdauungstrakt gelangende Phosphatasengemisch während der Passage nicht unverändert bleibt.

\section{The alkaline phosphatases in buman faeces}

The alkaline phosphatases in the faeces of healthy individuals and patients with intestinal illnesses were studied. It was shown by starch gel electrophoresis, measurements of total activitiy, inhibition by L-phenylalanine according to FISHMAN and Gosk (7) and heat inactivation at $56^{\circ}$ according to POSEN (6), that the alkaline phosphatases originate partly from the intestinal mucosa and partly from the bile. Phosphatases of other origin are also possibly present.

In many intestinal illnesses there are characteristic changes in the total excretion and in the ratio of the enzyme fractions. In enterocolitis and bacillary dysentery the faecal excretion of alkaline phosphatases was much higher than in healthy persons. In amoebiasis and lambliasis, the phosphatase excretion was decreased and the isoenzyme pattern and the degrees of inhibition and inactivation indicated that the excreted phosphatases were predominantly of biliary origin.

Alkaline phosphatases are probably partly degraded or inactivated during passage through the gut.

Die Enzyme des Verdauungstrakts kann man in zwei Gruppen einteilen: Verdauungsenzyme und Transportoder Resorptionsenzyme. Die Physiologie und Pathologie der Verdauungsenzyme ist weitaus besser bekannt als die der Enzyme mit Transportfunktion. Besonders ist das Verhalten dieser wichtigen Katalysatoren in Krankheitsfällen noch nicht näher erforscht.

Unter den Resorptionsenzymen nimmt die alkalische Phosphatase der Epithelzellen der Darmschleimhaut anscheinend eine wichtige Stellung ein. Auf ihre Teilnahme an der Fettresorption weist unter anderem der Anstieg dieser Phosphatase in der Lymphe und im Serum nach fetthaltigen Mahlzeiten hin, welche von LANGMANN und Mitarbeitern (1), WARNOCK (2) und KLEIN und Mitarbeitern $(3,4)$ beschrieben worden ist. Es ist bekannt, daß gesunde Erwachsene täglich viele Einheiten alkalischer Phosphatase im Stuhl ausscheiden. $\mathrm{Da}$ auch im Mekonium alkalische Phosphatase gefunden wurde (5) und die Exkretion durch per os applizierte Antibiotika nicht gehemmt wird, dagegen Immunserum gegen alkalische Phosphatase aus der Dünndarmschleimhaut die Aktivität der Faecesphosphatase inhibiert, hat Posen (6) die Meinung geäußert, daß die alkalische Phosphatase in den Exkrementen aus abgestoßenen Darmschleimhautzellen stammt. Die Faecesphosphatase wäre also vermutlich identisch mit dem intestinalen Isoenzym der Serumphosphatase.

In den Darm gelangen aber außer der alkalischen Schleimhautphosphatase auch Phosphatasen aus anderen
Organen, z. B. mit der Galle. Da Serumproteine in den Darm ausgeschieden werden, ist mit der Möglichkeit zu rechnen, daß in den Faeces alkalische Phosphatase vorkommen könnte, die aus den Knochen stammt. Alkalische Faecesphosphatasen könnten auch Produkte der Darmflora sein. Es kann daher vorausgesetzt werden, $\mathrm{da}$ in den Faeces mehrere alkalische Phosphatasen vorkommen. Erkrankungen der Organe, aus denen sie stammen, z. B. der Darmschleimhaut, könnten zu quantitativen und vielleicht auch qualitativen Änderungen im Spektrum der Ausscheidung führen.

Ausgehend von dieser Annahme untersuchten wir zunächst Faeces von gesunden Personen auf den Gehalt an alkalischer Phosphatase und versuchten mittels Stärkegelelektrophorese, durch Hemmung mit L-Phenylalanin nach FrshMAN und GHOSH (7) und durch Wärmeinaktivierung nach PosEN (8) Isoenzyme nach ihrer Herkunft zu unterscheiden. Auf die gleiche Weise analysierten wir Faeces von Darmkranken, Proben von Dünndarmschleimhaut, Blasengalle, Duodenalsaft, Kulturflüssigkeit von $E$. coli, sowie zum Vergleich auch normale und pathologische Sera.

\section{Untersuchungsmaterial}

Insgesamt wurden 67 Faecesproben analysiert; davon 46 mittels Stärkegelelektrophorese. Elf Proben stammten von in jeder Beziehung beschwerdefreien Erwachsenen; die übrigen von Patienten - Erwachsenen und Kindern - welche sich wegen chronischer Enterocolitis, Helminthiasis, Protozoasis oder Bakteriendysenterie in ärztlicher Behandlung befanden. 
Je $2 \mathrm{~g}$ frische Facces wurden mit $8 \mathrm{~m} /$ physiol. $\mathrm{NaCl}$-Lösung in der Reibschale homogenisicrt, die Suspension während $30 \mathrm{Min}$. mit etwa $500 \mathrm{~g}$ zentrifugiert, der abgegossene Extrakt mit physiol. $\mathrm{NaCl}-L o ̈ s u n g ~ z e h n f a c h$ verdünnt und sofort untersucht (Verdünnung der Facess 1:50).

Dünndarmschlcimhaut (4 Proben) und Blasengalle (6 Proben) stammten von frischen Leichen, bei welchen weder in vivo noch bei der Autopsic Zeichen von Erkrankungen der Verdauungsorgane nachgewiesen worden waren. Galle wurde durch Punktion der Gallenblase entnommen und unverdünnt sobald als möglich untersucht. Der bei der Autopsie entnommene Teil des Dünndarms (Jejunum) wurde sofort bei $-18^{\circ}$ eingefroren. Spätestens nach zwei Tagen wurde dic Schleimhaut der Darminnenfläche abgeschabt, gewogen und unter Zusatz der gleichen Menge physiol. $\mathrm{NaCl}$-Lösung $5 \mathrm{Min}$. im Apparat von Potrer und Elvenjem homogenisiert, das Homogenat $30 \mathrm{Min}$. mit etwa $500 \mathrm{~g}$ \%entrifugiert, der Uberstand abgegossen und sofort analysiert (Verdünnung der Darmschleimhaut $1: 2$ ).

Duodenalsäfte (25 Proben von cbensovielen Patienten mit chronischer Enterocolitis) wurden auf die übliche Weise mittels Duodenalsonde gewonnen, meist zwecks Untersuchung auf Lamblien. Alle Analysen wurden mit frischen, unverdünnten Säften angesetzt. Filtrate von zwei Tage alten Kulturen von $E$. coli untersuchten wir ohne weitere Vorbercitung. Da sie nur geringe Spuren von Phosphatasenaktivität zeigten, haben wir uns nicht weiter mit ihnen beschäftigt.

Serum wurde von nüchtern entnommenem frischem Venenblut sofort nach der Gerinnung abgetrennt und am selben Tag untersucht.

\section{Untersuchungsmethoden}

Von jedem Stuhl wurde eine abgewogene Portion bei $100^{c}$ zur Gewichtskonstanz getrocknet.

Die gesamte alkalische Phosphatasenaktivität wurde nach KIRBERGER und MARTINI (9) mit Dinatrium-monophenylphosphat bei pH 9,0 bestimmt. Alle Resultate sind in King-Armstrong-Einheiten angegeben.

Der Grad der Inhibition durch L-Phenylalanin wurde in parallelen Ansätzen gemessen, in denen dem Substrat $5 \mathrm{mM}$ dicser Aminosäure zugesetzt war. Er ist in Prozenten von der Gesamtaktivität ohne Inhibitor angegeben.

Zur Wärmeinaktivierung wurden aliquote Teile der Untersuchungsflüssigkeiten $15 \mathrm{Min}$. lang im Wasserbad auf $56^{\circ}$ erwärmt und die Restaktivität nach schnellem Abkühlen bestimmt. Der Aktivitätsverlust ist ebenfalls in Prozenten von der Gesamtaktivität ausgedrückt.

Zur Auftrennung der Isoenzymfraktionen diente horizontale Stärkegelelektrophorese nach SMrthirs (10), wobei nach Poulik (11) das Gel mit Tris-Citratpuffer; pH 8,65, bereitet war (Stärke der Connaught Laboratories), während die Küvetten mit Boratpuffer, $\mathrm{pH} 8,6$, gefüllt waren. In die Gelspalten wurde $0,02-0,03 \mathrm{ml}$ Untersuchungsflüssigkeit eingefüllt. Die Elektrophorese dauerte $6 \mathrm{Stdn}$. bei $6 \mathrm{~V} / \mathrm{cm}$ und $15-20^{\circ}$. Die Albuminfront der Serumproteine war dann $10 \mathrm{~cm}$ von der Auftragstelle entfernt.

Nach Beendigung des Laufs wurden die Phosphatasen in halbierten Gelstreifen nach BAMFord (12), mit $\alpha$-Naphtylphosphat und Fast Blue RR in Boratpuffer, pH 9,7, sichtbar gemacht. In einigen Fällen wurde die andere Hälfte des Gels mit Substratlösung entwickelt, welche $5 \mathrm{~mm}$ Phenylalanin enthielt.

Bei jeder Elektrophorese lief zwecks Lokalisation der Enzymfraktionen ein Elektropherogramm von Serum mit, von welchem eine Hälfte mit Amidoschwarz B auf Proteine und die andere auf Phosphatasen gefärbt wurde. Die in den verschiedenen Arten von Untersuchungsmaterial gefundenen Phosphatasenfraktionen sind durchwegs nach ihrer Lage in bezug auf die Serumproteine bezeichnet.

\section{Ergebnisse der Elektrophorese}

Auf der Abbildung 1 sind Aufnahmen einiger Elektropherogramme zusammengestellt. Nur die anodische
$\Theta$




Abb. 1

Alkalische Phosphatasen in Stärkegelelektropherogrammen von verschiedenem biologischen Material

a) Serumproteine. b)-h) Phosphatasen. b) Normalserum. c) Blasengalle. d) Duodenalsaft. e) Extrakt aus Dünndarmschleimhaut. f) Faeces (Dysenterie). g) Faeces (ohne Beschwerden). h) Faeces (Amoebiasis)

Seite ist gezeigt, weil kathodisch niemals Phosphatasen gefunden worden sind. Die Wanderungsgeschwindigkeit war in allen Fällen die gleiche.

Streifen a und b sind Elektropherogramme eines normalen Erwachsenenserums. a ist mit Amidoschwarz gefärbt, in $b$ sind nach BAMFord Phosphatasen dargestellt. Auf b ist, wie meist in Normalserum von Erwachsenen, nur eine Phosphatasenfraktion im Bereich $\operatorname{der} \beta$-Globuline zu sehen. In Kinderserum kam regelmäßig neben der $\beta$-Globulinfraktion eine etwas langsamere zum Vorschein, von der man annimmt, daß sie aus den Knochen stammt. Im Serum von Kranken mit Lebercirrhose wurde die noch etwas langsamere sogenannte ,intestinale“ Fraktion im Bereich der Haptoglobine gefunden.

Streifen c zeigt, daß auf Elektropherogrammen von Blasengalle mehrere Phosphatasenfraktionen vorkommen können. Es sind mehrere Banden sichtbar, welche recht verschiedene Beweglichkeiten hatten. Charakteristisch für Galle scheinen die schnellen Fraktionen zu sein die mit den Albuminen oder gleich hinter ihnen wandern, sowie die langsamen, in der Nähe des Starts zurückbleibenden. 
Streifen d stammt von Duodenalsaft. Es sind zweif. Fraktionen zu sehen, eine im Bereich der Haptoglobine, die zweite in der Nähe des Starts. In anderen Duodenal säften wurden schnell wandernde Fraktionen gefunden wie auf Elektropherogrammen von Galle.

Auf e ist ein hochaktiver Extrakt aus Dünndarmschleimhaut aufgetrennt. Bei weitem der größte Teil der Aktivität befindet sich im Bereich der Haptoglobine und $\beta$ Globuline. Die beiden schwachen langsamen Banden entsprechen den langsamen Banden auf dem Gallenelektropherogramm c; in anderen Dünndarmextrakten kamen auch schnelle Fraktionen vor, ebenfalls ähnlich denen in der Galle.

Unter den drei Faecesextrakten zeichnet sich h, welcher von einem Patienten mit Amoebiasis stammte, dadurch aus, da $B$ er nur langsame Fraktionen zeigt, während sich auf $f$ und $g$ die Hauptaktivität im Bereich $\operatorname{der} \beta$-Globuline befindet.

Streifen $f$ stammte von einem Patienten mit schwerer Bakteriendysenterie und $g$ von einer gesunden Person. Sie unterscheiden sich hauptsächlich durch die Intensität der Hauptfraktion. Entwicklung dieser Elektropherogramme in Gegenwart von L-Phenylalanin zeigte, daß die Hauptfraktion durch Phenylalanin stark inhibiert wurde, wobei zugleich zum Vorschein kam, daß sie aus zwei oder drei dicht nebeneinander wandernden Banden bestand. Abbildung 2 zeigt dieses Verhalten.

$\Theta$



Abb. 2

Hemmung alkalischer Faecesphosphatase durch L-Phenylalanin

Abbildung 3 ist eine schematische Zusammenfassung aller in Galle, Darmschleimhaut, Duodenalsaft und Faeces gefundenen Enzymfraktionen. Zum Vergleich sind die Serumproteine und die in normalem und pathologischem Serum gefundenen Banden mit eingezeichnet. Es ist ersichtlich, $\mathrm{da} B$ in allen untersuchten, aus dem Verdauungstrakt stammenden Arten von Material drei Gruppen von Phosphatasenfraktionen gefunden worden sind. Der größte Teil der Aktivität befand sich meist im Bereich der $\beta$-Globuline und Haptoglobine und zeichnete sich in zwei oder drei Banden ab, welche wir „mittlere“ nennen möchten. An dieser Stelle befinden sich auch die wichtigsten Isoenzyme der alkalischen Serumphosphatase. In normalem Serum kommt regelmäßig die schnellste der drei mittleren Fraktionen vor; gleich hinter ihr findet man die sogenannte „Knochenphosphatase“ die im Serum von Kindern fast immer deutlich zu sehen ist. Noch etwas langsamer wandert die ,intestinale“ Phosphatase, welche wir nur in Fällen von Lebercirrhose gesehen haben. In Fáecesextrakten und anderem Material aus dem Verdauungstrakt war niemals eine Komponente mit der Beweglichkeit der Knochenphosphatase zu sehen.

Eine zweite Gruppe von „schnelleren“ Komponenten kam in Galle, Duodenalsaft und Extrakten aus Darmschleimhaut vor, ist aber niemals in Stuhlextrakten gefunden worden.

Eine dritte, „langsamere“ Gruppe bestand meist aus zwei oder drei Komponenten, von denen eine fast an der Ausgangstelle zurückblieb. In manchen Faeces war außerdem ein starkes Band im Bereich der $\beta$-Lipoproteine zu sehen wie auf Band h der Abbildung 1.

Die auf der Abbildung 3 resümierten Enzymfraktionen waren nur selten alle zusammen in derselben Probe zu sehen. Elektrophoresen von 25 Duodenalsäften zeigten z. B. mehrere Muster; auf der Abbildung 4 ist angegeben, wie oft die einzelnen Fraktionen gefunden worden sind.

Abbildung 4 zeigt, da $\mathrm{B}$ die drei mittleren Fraktionen in Duodenalsäften nie zusammen gefunden worden sind; am häufigșten kam nur eine von ihnen zum Vorschein, und zwar die langsamste.

Auf der Abbildung 5 sind auf die gleiche Weise schematisch Elektropherogramme von Phosphatasen in' Faecesextrakten gesunder und kranker Personen dargestellt; zugleich ist die Häufigkeit der Konstellationen angegeben.

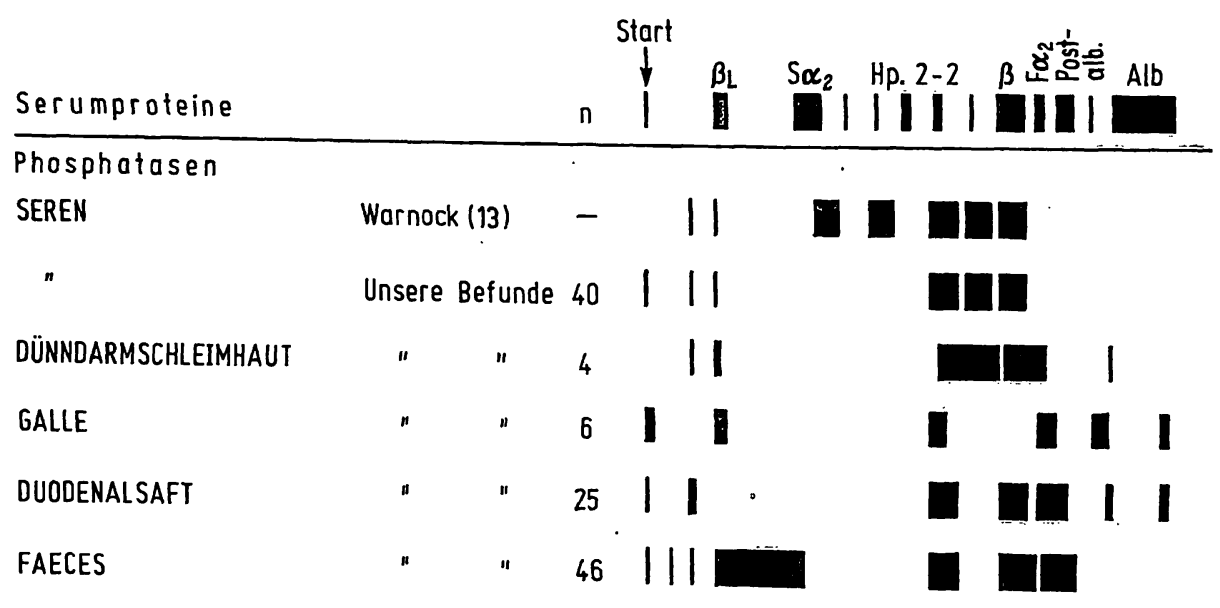

Abb. 3

Vergleich der Phospatasenfraktionen in Stärkegelelektropherogrammen 


Serumproteine |
$\begin{aligned} & \text { Phosphatasen- } \mid \\ & \text { banden }\end{aligned}$

Insgesamt nach- 912 gewiesen in

$\begin{array}{llllll}17 & 5 & 5 & 1 & 7 & \text { Proben }\end{array}$

Abb. 4

Frequenz der Phosphatasenbanden in Stärkegelelektropherogrammen von 25 Duodenalsäften

Auf der Abbildung 5 ist zu sehen, daß die mittleren Fraktionen in den Stühlen von Gesunden und Kranken vorherrschten. Aber in Fällen von Gastroenteritis fehlte in der mittleren Gruppe stets die langsamste, bei den Haptoglobinen liegende Bande, welche auch bei $\mathrm{Ge}$ sunden nicht immer bemerkbar war.

Alle drei mittleren Banden fehlten in den meisten Fällen von Protozoasis, in welchen sich dagegen der größte Teil der Aktivität im Bereich der $\beta$-Lipoproteine befand (s. Abb. $1 \mathrm{im}$ Band h). Die Verschiebung der Aktivität von der mittleren Gruppe zur langsamen $\beta$-Lipoproteinfraktion schien in solchem $\mathrm{Maße}$ charakteristisch für Protozoeninfektion, $\mathrm{da} B$ auf Grund eines solchen Befundes bei einer angeblich darmgesunden Person eine genaue Stuhluntersuchung ausgeführt wurde, wobei man Entamoeba bistolytica entdeckte.

Bei erfolgreicher Behandlung von Parasitenerkrankungen verschwand die $\beta$-Lipoproteinfraktion und wurde durch die im Stuhl von Gesunden vorkommenden mittleren Fraktionen ersetzt. Abbildung 6 zeigt diese Änderung des Elektrophoresebildes in zwei Fällen.

Bei der Beurteilung der Resultate der Elektrophorese muß man in Betracht ziehen, daß die Gesamtaktivität in den aufgetragenen Volumina von $0,02-0,03 \mathrm{~m} /$ bei verschiedenen Arten von Untersuchungsmaterial sehr verschieden war. In KA Einheiten ausgedrückt befanden sich auf den Elektropherogrammen ungefähr folgende Gesamtmengen von alkalischer Phosphatase:

Aus Faeces gesunder Personen etwa 0,001 -0,015

Aus Duodenalsaft etwa 0,0003-0,05

Aus Galle etwa $0,002-0,2$

Aus Dünndarmschleimhaut etwa $0,4 \quad-0,9$

Die Verschiedenheiten zwischen Elektropherogrammen einzelner Proben gleichartigen Materials sind daher vermutlich zum Teil darauf zurückzuführen, daß schwä-



J.M. 15. IV. 1968

" 9. D. 1969



Ånderung der Phosphatasenfraktionen in Faecesextrakten bei erfolgreicher Behandlung von Protozoasis/M. M.: Entamoeba histolytica, J. M.: Lamblia intestinalis

chere Fraktionen nicht sichtbar wurden, wenn die aufgetragene Enzymmenge sehr klein war. Die größten Chancen; alle vorhandenen löslichen Fraktionen zur Darstellung zu bringen, bestanden bei Analysen von Dünndarmschleimhaut.

\section{Ergebnisse der quantitativen Analysen}

In der Tabelle 1 sind extreme Befunde der Gesamtaktivität und des Grads der Inhibition durch $5 \mathrm{~mm}$ L-Phenylalanin von alkalischen Phosphatasen in Duodenalsaft, Blasengalle, Extrakten von Dünndarmschleimhaut
Abb. 5

Alkalische Phosphatasenbanden in Stärkegelelektropherogrammen von Faecesextrakten gesunder und kranker Personen

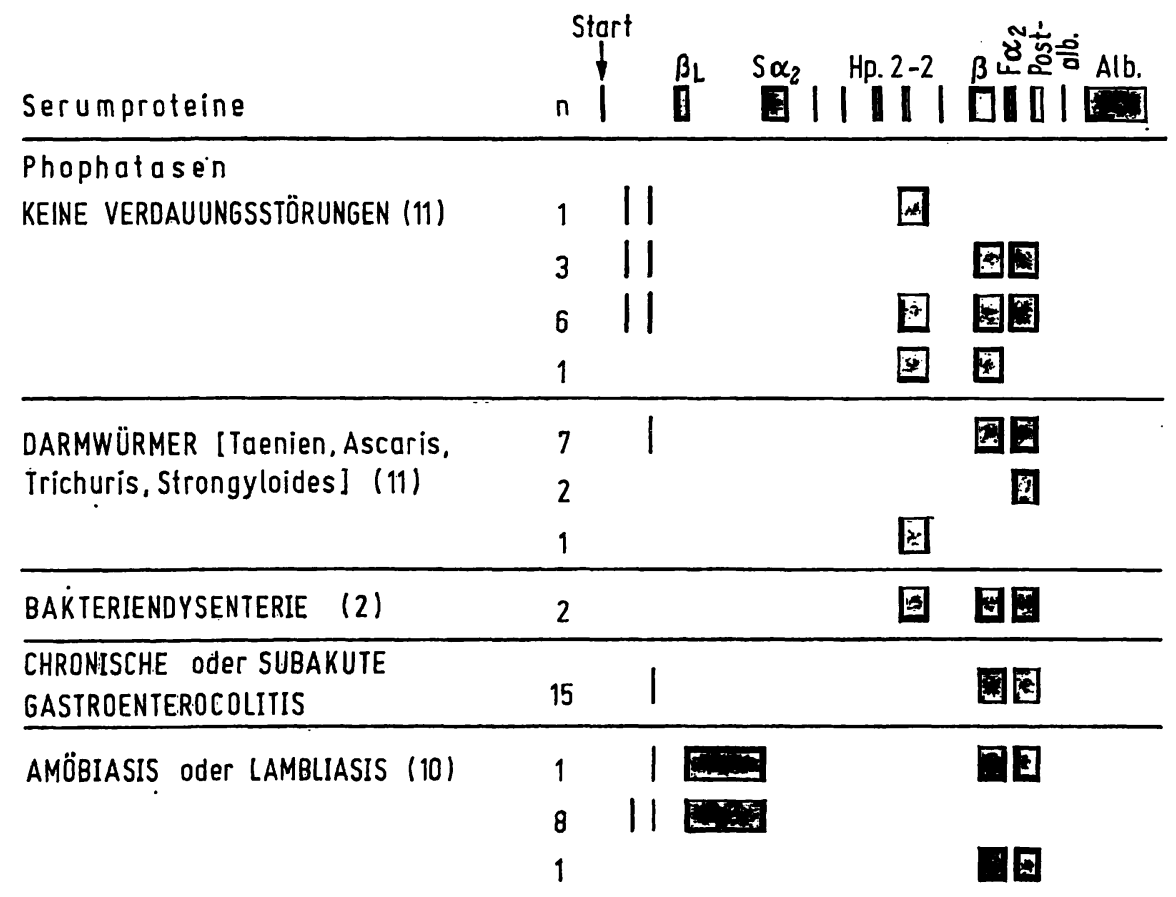


Tab. 1

Gesamtaktivität und Hemmung durch L-Phenylalanin alkalischer Phosphatasen in Duodenalsaft, Blasengalle, Dünndarmschleimhaut und Faeces gesunder Personen

\begin{tabular}{|c|c|c|c|c|}
\hline \multirow[b]{2}{*}{ Material } & \multirow[b]{2}{*}{$\begin{array}{l}\text { Zahl der } \\
\text { Proben }\end{array}$} & \multirow[b]{2}{*}{$\begin{array}{c}\text { Gesamtaktivität } \\
\text { KA Einheiten }\end{array}$} & \multicolumn{2}{|c|}{ Abnahme der Aktivität (\%) } \\
\hline & & & $\begin{array}{c}\text { in Gegenwart von } 5 \mathrm{~mm} \\
\text { L-Phenylalanin }\end{array}$ & $\begin{array}{c}\text { beim Erwärmen auf } 56^{\circ} \\
\text { während } 15 \text { Min. }\end{array}$ \\
\hline Blasengalle & 6 & $\begin{array}{c}0,09-9,5 \\
\text { pro ml }\end{array}$ & $6,5-29,3$ & \\
\hline Duodenalsaft & 25 & $\begin{array}{c}0,015-1,68 \\
\text { pro ml }\end{array}$ & $50,0-91,8$ & \\
\hline Schleimhautextrakt & 4 & $\begin{array}{c}41,0-89,4 \\
\text { pro } \mathrm{g} \text { Schleimhaut }\end{array}$ & $66,6-77,0$ & \\
\hline $\begin{array}{c}\text { Faeces gesunder } \\
\text { Erwachsener }\end{array}$ & 11 & $\begin{array}{l}2,3-30,0 \\
\text { pro } g \text { frischen Faeces }\end{array}$ & $46,0-91,1$ & $0-55,2$ \\
\hline
\end{tabular}

Tab. 2

Alkalische Phosphatasenaktivität in Faeces von Gesunden und Darmkranken. $\overline{\mathbf{x}} \pm s_{\overline{\mathbf{x}}}$

\begin{tabular}{|c|c|c|c|c|c|}
\hline Diagnose & $\begin{array}{c}\text { Zahl der } \\
\text { Proben }\end{array}$ & $\begin{array}{c}\text { Trockensubstanz } \\
\%\end{array}$ & $\begin{array}{c}\text { Gesamtaktivität } \\
\text { KA Einheiten } \\
\text { pro g Trockensubstanz }\end{array}$ & $\begin{array}{l}\text { Abnahme der } \\
\text { in Gegenwart von } 5 \mathrm{~mm} \\
\text { L-Phenylalanin }\end{array}$ & $\begin{array}{l}\text { Aktivität (\%) } \\
\text { beim Erwärmen auf } 56^{\circ} \\
\text { während } 15 \text { Min. }\end{array}$ \\
\hline Gesund & 11 & $23,1 \pm 0,65$ & $82,4 \pm 0,51$ & $69,0 \pm 1,8$ & $14,3 \pm 1,6$ \\
\hline Chronische Enterocolitis & 10 & $16,0 \pm 0,50$ & $334,9 \pm 3,77$ & $69,6 \pm 1,8$ & $39,4 \pm 2,5$ \\
\hline Helminthiasis*) & 15 & $17,5 \pm 0,59$ & $108,6 \pm 0,70$ & $50,4 \pm 2,1$ & $36,7 \pm 1,6$ \\
\hline Protozoasis**) & 17 & $18,6 \pm 0,47$ & $48,4 \pm 0,25$ & $47,3 \pm 1,6$ & $25,5 \pm 1,1$ \\
\hline $\begin{array}{l}\text { Protozoasis mit } \\
\beta \text {-Lipoproteinfraktion } * * * \text { ) }\end{array}$ & 12 & $14,9 \pm 0,45$ & $68,5 \pm 0,25$ & $34,8 \pm 1,9$ & $18,8 \pm 0,9$ \\
\hline Bakteriendysenterie****) & $\begin{array}{l}1 \\
1\end{array}$ & $\begin{array}{l}10,2 \\
26,6\end{array}$ & $\begin{array}{r}2115 \\
780\end{array}$ & $\begin{array}{l}79,3 \\
68,2\end{array}$ & $\begin{array}{l}7,4 \\
4,7\end{array}$ \\
\hline
\end{tabular}

*) Ascaris, Trichuris, Strongyloides, Taenia saginata

**) Lamblia intestinalis, Entamoeba histolytica

***) Fälle, in denen die im Bereich der $\beta$-Lipoproteine wandernde Fraktion nachgewiesen war ****) Shigella FLEXNER

Tab. 3

Signifikanz der Differenzen zwischen Durchschnittswerten fû̀r Phosphatasen im Stuhl von Gesunden und Kranken. Zahl der Fälle wie in Tab. 2



n. s. = nicht signifikant

und Faeces von gesunden Erwachsenen resümiert; für Faeces ist auch der Grad der Inaktivierung bei $56^{\circ}$ angegeben.

In der Tabelle 2 sind durchschnittliche Resultate $\left(\bar{x} \pm s_{\bar{x}}\right)$ von Faecesanalysen angeführt.

Die in der Tabelle 2 angeführten Daten zeigen, daß in bezug auf die Phosphatasenexkretion zwischen Gesunden und Kranken große Unterschiede bestanden. Patienten mit Enterocolitis schieden im Durchschnitt viel mehr, Protozoenträger aber weniger alkalische Phosphatase aus als Gesunde. Besonders hohe Werte haben wir in zwei Fällen von Bakteriendysenterie gefunden. Die Aktivität in den Stühlen von Gesunden schwankte allerdings in weiten Grenzen $(s=5,54)$, sehr viel größer war aber die Streuung der Einzelwerte bei Kranken mit Enterocolitis $(s=37,69)$ und bei Helminthenträgern $(s=10,41)$, während sie bei Protozoasis etwas kleiner war $(s=4,28)$, als bei Gesunden.

Große Unterschiede bestanden auch in der Empfindlichkeit der Faecesphosphatasen gegenüber I-Phenyl- alanin und erhöhter Temperatur. Die Tabelle 3 zeigt, welche von den beobachteten Differenzen zwischen Durchschnittswerten bei Gesunden und Kranken statistisch signifikant waren.

Wenn man die gegensinnig von den Normalwerten abweichenden Durchschnittswerte der Gesamtaktivität und der Inhibition durch I-Phenylalanin bei Enterocolitis und Protozoasis vergleicht, zeigt es sich, daß die Unterschiede zwischen diesen beiden Gruppen hochsignifikant waren (für Gesamtaktivität $P<0,001$, für Hemmung durch $\mathrm{L}$-Phenylalanin $\mathrm{P}<0,01)$.

\section{Diskussion}

Fishman und Grosh (7) haben die Wirkung von LPhenylalanin auf humane alkalische Phosphatasen aus verschiedenen Organen gemessen und Posen (8) hat den Aktivitätsverlust beim Erwärmen bestimmt. Die Resultate dieser Autoren, zusammen mit einigen eigenen Beobachtungen sind in der Tabelle 4 zusammengefaßt. Auf der Abbildung 7 ist das Verhältnis zwischen der 
Tab. 4

Inhibition alkalischer Phosphatasen aus verschiedenen Organen durch $5 \mathrm{~mm}$ L-Phenylalanin und Inaktivierung durch Erwärmen auf $56^{\circ}$ während 15 Min. Abfall der Aktivität in Prozenten der Gesamtaktivität

\begin{tabular}{|c|c|c|c|c|c|}
\hline & \multicolumn{5}{|c|}{ Aktivitätsverlust (in \%) der alkalischen Phosphatasen } \\
\hline & Knochen & Leber & Galle & $\begin{array}{l}\text { Duodenal- } \\
\text { saft }\end{array}$ & $\begin{array}{l}\text { Dünndarm- } \\
\text { schleimhaut }\end{array}$ \\
\hline $\begin{array}{l}\text { Hemmung durch } 5 \text { mM L-Phenylalanin } \\
\text { nach FISHMAN und GHOSH (7) }\end{array}$ & $10,3 \pm 6,3$ & $7,7 \pm 1,9$ & $9,0 \pm 2,4$ & $81,0 \pm 1,0$ & $77,0 \pm 2,8$ \\
\hline $\begin{array}{l}\text { Hemmung durch } 5 \mathrm{~mm} \text { L-Phenylalanin } \\
\text { eigene Beobachtungen }\end{array}$ & & & $6,5-29,3$ & $50,0-91,8$ & $66,6-77,0$ \\
\hline $\begin{array}{l}\text { Inaktivierung bei } 56^{\circ} \text { während } 15 \text { Min. } \\
\text { nach POSEN (8) }\end{array}$ & 81,2 & 75,2 & 35,0 & & 68,1 \\
\hline
\end{tabular}



Abb. 7

Verhältnis zwischen Inhibition von Phosphatasen in Faecesextrakten durch $5 \mathrm{~mm}$ L-Phenylalanin und Inaktivierung durch 15 Min. dauerndes Erwärmen auf $56^{\circ}$. Gesunde, o Darmkranke (Enterocolitis, Bakteriendysenterie, Protozoasis)

Inhibition der Phosphatasen durch I-Phenylalanin und der Inaktivierung durch 15 Min. langes Erwärmen auf $56^{\circ}$ in jenen Stuhlextrakten, welche gleichzeitig auf beide Arten geprüft worden waren, graphisch dargestellt.

Es zeichnen sich auf dieser Abbildung in bezug auf Inhibition und Inaktivierung drei Kombinationen ab. In einem Teil der Fälle wurde die Aktivität durch LPhenglalanin um 10-40\% und durch Erwärmen um höchstens 33\% herabgesetzt. In den meisten Extrakten fiel die Aktivität in Gegenwart von L-Phenylalanin um 50-90\%, wurde aber durch Erwärmen auch nur. um ein Drittel verringert. Nur in einer kleineren Zahl von Extrakten waren die Phosphatasen sowohl gegen L-Phenylalanin als auch gegen Erwärmen hochempfindlich.

Gallenphosphatase ist verhältnismäßig resistent gegen Inhibition und Inaktivierung. In den schwach inhibierten und wenig inaktivierten Fällen auf der linken Seite der Abbildung 7 scheinen in den Faeces vorwiegend Gallenphosphatasen enthalten gewesen zu sein. Alle diese Extrakte stammten von Kranken mit Protozoasis oder Helminthiasis; in den meisten war auch bei der Elektrophorese nur die beschriebene langsame $\beta$-Lipoproteinfraktion gefunden worden. Das ist ein Grund mehr für die Annahme, daß diese Fraktion aus der Galle stammt.

Alkalische Phosphatase aus Dünndarmschleimhaut wird sowohl stark inhibiert als auch hochgradig inaktiviert. In den Fällen, in welchen Inhibtion und Inaktivierung beide sehr beträchtlich waren, schienen in den Faeces hauptsächlich Dünndarmphosphatasen vorhanden $z \mathrm{u}$ sein. In dieser Gruppe befanden sich einzelne Fälle von Enterocolitis und Helminthiasis und auch ein Extrakt aus dem Stuhl einer gesunden Person. Auf den Elektropherogrammen dieser Stuhlextrakte war die Phosphatasenaktivität im Bereich der beiden schnelleren mittleren Fraktionen konzentriert, welche auch in Extrakten aus Dünndarmschleimhaut die stärksten waren und bei Entwicklung der Elektropherogramme in Gegenwart von L-Phenylalanin sehr abgeschwächt wurden (s. Abb. 2). In der durch L-Phenylalanin stark inhibierten, aber durch Erwärmen nur wenig inaktivierten Gruppe befanden sich fast alle Extrakte aus Faeces von Gesunden (10 von 11 untersuchten Proben) sowie aus Faeces von Kranken mit Enterocolitis, Bakteriendysenterie und einige Fälle von Helminthiasis und Protozoasis. Keine der in der Tabelle 4 angeführten Phosphatasen entspricht diesem Typus. Elektrophoresebilder zeigten zwar in diesen Fällen die stärksten Enzymbanden an den Stellen der Dünndarmphosphatasen, aber Inhibition und Inaktivierung sprechen dafür, daß auch andere, beim Erwärmen besonders stabile, vermutlich aus der Galle stammende Komponenten anwesend waren, welche bei der Elektrophorese in Stärkegel die gleiche Beweglichkeit hatten wie Dünndarmphosphatasen.

Vergleich der Elektropherogramme von Duodenalsaft, Dünndarmschleimhaut, Galle und Faeces führen zu dem Schluß, daß zumindest einzelne Phosphatasen Komponenten während der Passage durch den Verdauungstrakt teilweise verändert, inaktiviert oder abgebaut werden. Dafür spricht u. a. die vollkommene Abwesenheit der schnellen Fraktionen in Faecesextrakten. In diesem Sinn spricht auch eine einfache Überschlagrechnung.

Nach Bodansky (14) wiegen frische Faeces Gesunder 60-250 g pro Tag, im Durchschnitt $150 \mathrm{~g}$. Bei einem durchschnittlichen Gehalt an Trockensubstanz von $23 \%$ und einer durchschnittlichen Gesamtaktivität pro $g$ Trockensubstanz von $82 \mathrm{KA}$ Einheiten (s. Tab. 2) würden gesunde Erwachsene pro Tag etwa $2750 \mathrm{KA}$ Einheiten alkalische Phosphatase ausscheiden.

Bei einem täglichen Gallenfluß von nur $500 \mathrm{ml}$ pro Tag und einer minimalen Phosphatasenaktivität der Galle von nur $9 \mathrm{KA}$ Einheiten (s. Tab. 1) würden täglich mit der Galle allein $5400 \mathrm{KA}$ Einheiten alkalische Phosphatase in den Darm gelangen, d. h. mehr als in den Faeces ausgeschieden werden. 


\section{Literatur}

1. Langman, M. J. S., E. Leuthold, E. B. Robson. J. Harris, J. E. Luffman und H. Harris, Nature (London) 212, 41 (1966). 2. Warnock, M. L., Proc. Soc. exper. Biol. Med. 129, 768 (1968). -3. Klein, U. E., H. Ch. Drube, H. Th. Hansen und F. Mielke, Klin. Wschr. 45, 244 (1967). - 4. KLEIN, U. E., Dtsch. med. Wschr. 94, 526 (1969). - 5. DAFLQVIST, A. und T. LINDBERG, Clin. Sc., London 30, 517 (1966). - 6. Posen, S., Ann. Internal Med. 67, 183 (1967). - 7. Fishman, W. H. und N. K. Grosh, Adv. Clin. Chem. 10, 255 (1967). - 8. Posen, S., F. C. NeALE und J. S.
Clubb, Ann. Internal Med. 62, 1234 (1965). - 9. KIrberger, E. und G. A. Martini, Dtsch. Arch. klin. Med. 197, 268 (1950). 10. Smithies, O., Biochem. J. 61, 629 (1955). - 11. Poulik, M. D., Nature (London) 180, 1477 (1957). - 12. BAmFord, K. F., H. Harris, J. E. Luffman, E. B. Robson und T. E. Cleghorn, Lancet, London 1965/I, 530. - 13. WARnoCK, M. L., Clin. Chim. Acta (Amsterdam) 14, 156 (1966). - 14. Bodanskx, M. und O. Bodanskr, Biochemistry of Disease, 2. Aufl. S. 348, The Mac Millan Co., New York (1952).

Frau Prof.

Dr. Marijana Fišer-Herman Bijenička 21

Zagreb, Jugoslawien 